\title{
Optimal traffic calming: A mixed-integer bi-level programming model for locating sidewalks and crosswalks in a multimodal transportation network to maximize pedestrians' safety and network usability
}

\begin{abstract}
We study the effect that installing sidewalks and crosswalks, as traffic calming facilities, has on the safety and usability of a transportation network with automobile, public transit and walking as modes of transportation. A mathematical programming model is proposed for this problem whose objective is to minimize the safety hazard for pedestrians and the total transportation cost of the network. We utilize a customized greedy heuristic and a simulated annealing algorithm for solving the problem. The computational results indicate that installing sidewalks and crosswalks at proper locations can reduce the overall transportation cost and improve pedestrians' safety.
\end{abstract}

Keywords: Multimodal transportation network, mixed-integer nonlinear programming, network design, pedestrians' safety, traffic calming, user equilibrium.

\section{Introduction}

In this study, we use traffic calming facilities such as sidewalks and crosswalks (S\&C) as pedestrians' infrastructures in designing a multimodal transportation network to enhance pedestrians' safety and increase network usability. We consider walking as a main mode of transportation, besides automobiles and public transit, and pedestrians as one of main users of the transportation system.

\subsection{Motivation}

In many small communities in the United States, transportation is dominated by a single mode - the motor vehicle. The lack of walkway infrastructures like sidewalks and crosswalks poses a safety hazard to pedestrians since they must walk along busy streets and highways to travel within the city. Since the 1920s, there has been a growing concern about pedestrians' safety because pedestrian fatalities are a major part of all traffic fatalities (Campbell et al., 2004). Pedestrians' safety is defined as the condition of being protected from danger, risk, or injury caused by accidents with motor vehicles. The Federal Highway Administration (FHWA) estimated that 4,500 pedestrians are killed annually because of traffic accidents with motor vehicles, and as many as $88 \%$ of those accidents could have been avoided if walkways separate from travel lanes had been available to pedestrians (FHWA, 2010). This implies the importance of pedestrians' safety in transportation network design for city planners and government. On the other hand, pedestrians are also concerned about safety and consider it as an important factor in transportation (Bahari et al., 2013; Weinstein Agrawal et al., 2008). 
City planners use traffic calming measures to improve the conditions for non-motorized street users including pedestrians and to increase drivers' awareness of those users which can enhance pedestrians' safety (Lee et al., 2013; Nadesan-Reddy and Knight, 2013; Prokopich and Wise, 2014). Traffic calming is a combination of mainly physical measures that reduces the negative effects of motor vehicle use, alters user behavior and improves conditions for non-motorized travelers (FHWA and ITE, 1999). S\&C are among the most important and effective traffic calming measures for enhancing pedestrians' safety (Campbell et al., 2004). Carefully installed S\&C improve the walkability of transportation systems, which not only can enhance pedestrians' safety, but can also encourage more people to walk (Freeman et al., 2013; Friederichs et al., 2013; Gallimore et al., 2011) which results in reducing vehicle miles traveled, alleviating traffic congestion, cutting energy use and carbon emissions, and reducing noise and air pollution (Marshall and Garrick, 2010). However, considering the limited budget for city planners and the large number of possible alternatives for locating and installing S\&C, identifying the optimal location for these city infrastructures is an important and challenging issue. Therefore, developing a decision support tool that can help decision makers with identifying optimal locations for S\&C in a transportation network is needed.

\section{2. $\quad$ Related Literature}

The transportation network design problem (TNDP) has long been recognized as one of the most difficult and challenging problems in transportation and urban planning. The majority of research in TNDP literature focuses on developing mathematical formulations and solution techniques for improving the utilization of the transportation network through either link improvements (i.e. expanding the capacity) or link additions (e.g. building new streets) (Farahani et al., 2013). The common objective in TNDP is to make an optimal investment decision in order to minimize the total travel cost in the network.

The TNDP is usually formulated as a bilevel, leader-follower problem, (Farvaresh and Sepehri, 2013; Khooban et al., 2015; Szeto and Jiang, 2014; Yu et al., 2015). The upper level is the designers' problem in which decision makers (e.g. city planners) design the transportation network. The lower level problem is the travelers' problem in which travelers decide on their travel route and mode of transportation. The bilevel structure allows the decision maker to improve the transportation network while accounting for travelers’ route and mode decisions (Farahani et al., 2013).

Although there have been many studies of the TNDP, there are still numerous gaps and limitations in the literature such as the following:

(1) many transportation network design problems address only a single mode, and "the literature of multimodal network design problem is very limited” (Farahani et al., 2013). We found only one study 
in multimodal TNDP that considers a non-motorized mode (bicycle) (Seo et al., 2005); all other studies focus on either bus and car or on bus, car, and metro (Gallo et al., 2011; Miandoabchi et al., 2012, 2011b; Szeto et al., 2010). In pedestrian transportation literature, most studies are descriptive (Buehler and Pucher, 2012; Millward et al., 2013; Stewart et al., 2016; Weinstein Agrawal et al., 2008), and, to the best of our knowledge, no study has considered walking as a mode of transportation within a network design problem (even though, in everyday life, walking is actually the most important mode of transportation).

(2) Of the few existing studies in multimodal transportation problems, most of them assume no flow interaction between different transportation modes (Beltran et al., 2009; Cantarella et al., 2006; Fan and Machemehl, 2008). However, in reality when the transportation modes share lanes, the flow of different transportation modes do interact.

(3) Most studies in TNDP have ignored combined mode trips where travelers can use multiple modes of transportation during the course of their trips such as park-and-ride, especially in the strategic level decisions (Farahani et al., 2013). An important aspect of multimodal transportation systems with combined mode trips is to provide convenient mode transfer possibilities for travelers. Nowadays, with the advent of technologies like Uber, Lyft, SideCar and Curb, combined mode trips seem more viable than ever before.

(4) The objective in most studies in TNDP is primarily related to travel time (Mesbah et al., 2008; Resat and Turkay, 2015; Yao et al., 2014; Yu et al., 2015), or travel cost such as operator cost, user cost, etc., e.g. (Cipriani et al., 2006; Fan and Machemehl, 2008; Gallo et al., 2011); one of them addresses safety.

Given these gaps in the existing literature on the TNDP, there is a need for further study that investigates network design from a pedestrian perspective.

\subsection{Research scope and contributions}

In this paper, we study a TNDP that addresses all of the above mentioned gaps and limitations in the literature toward the goal of extending existing literature to make it applicable to pedestrian transportation. We propose a network design framework for a multimodal TDNP that addresses the aforementioned gaps in the literature: (1) It considers walking as an independent mode of transportation in addition to public transit (i.e. bus) and automobile. This means that pedestrians are considered as one of the main users of the transportation system. (2) It considers flow interactions between different modes of transportation (e.g. the flow of cars influence the flow of bus and vice versa). (3) It allows travelers to switch between different modes of transportation along the course of their journey (i.e. combined mode trips). (4) It considers pedestrians' safety as a major criterion in designing a transportation network. The 
goal of this study is to optimize the usability of the transportation system while ensuring safe travel for pedestrians by optimally locating S\&C.

We develop a bilevel mathematical programming model for optimally locating S\&C in a transportation network such that the overall transportation cost decreases and pedestrians' safety improves. To reflect the importance of safety for both city planners and pedestrians, we include safety both in the design level (upper level) and in the user level (lower level) of the model. We found that solving large instances of this problem using a commercial solver requires excessive computational resources. Therefore, we apply two heuristic algorithms, a customized greedy heuristic and a simulated annealing algorithm. We test these algorithms on three problem instances: the hypothetical Small network (a network with 4 nodes and 5 links), the Hearn network (Hearn and Ramana, 1998) and the Sioux Falls network (LeBlanc, 1975).

The major contribution of this research is developing a quantitative network design model for locating sidewalks and crosswalks in a transportation network to reduce the overall cost, improve pedestrians' safety and enhance walkability.

The remainder of the paper has the following outline: Section 2 describes the problem and presents the proposed mathematical model formulation for the problem; Section 3 discusses the solution methodologies for solving the problem; Section 4 conducts numerical experiments on three sample networks; Section 5 gives concluding remarks and discusses future research directions.

\section{Optimal Traffic Calming Implementation in a Multimodal Transportation Problem (OTCIMTP)}

The problem is, given a limited budget, to determine where to install traffic calming facilities (S\&C) in a transportation network, to minimize the total transportation cost, and to improve pedestrians' safety. For any traffic calming installation layout, the problem also includes identifying a traffic assignment which is in accordance with the user equilibrium for a multimodal transportation problem. In transportation network problems, user equilibrium is the situation in which no traveler believes that his/her travel time can be improved by unilaterally changing routes. In this research, travelers can use three modes of transportation: automobile, public transit and walking. Travelers are allowed to switch between these modes along their trips. We assume a traffic calming layout with the minimum transportation cost, and the minimum safety hazard for pedestrians, is the best traffic calming layout.

We formulate OTCIMTP as a bi-level, leader-follower optimization model. In the upper level, the leader (city planners) decides where to locate the traffic calming facilities, and in the lower level, the 
follower (travelers) decides on the travel route and mode of transportation (Farahani et al., 2013). The lower level problem is a traffic assignment problem under the user equilibrium.

We use a network reconstruction process to add S\&C to an existing transportation network dataset with only one mode, the automobile mode. Given the road transportation network dataset, this process adds walking and public transit modes as a set of mode-specific links and nodes to the transportation network. In this framework, pedestrians use walking links, automobile passengers use automobile links, and public transit passengers use public transit links. The reconstruction process is briefly discussed in the next section (for a more detailed discussion on the reconstruction process we refer the reader to Parsafard et al. (2015)).

The main assumptions of this study are as follows:

a) Since walking is considered as a mode of transportation, the travel demand is based on the number of travelers, not the number of automobiles or public transits. There are three different transportation users: the automobile passengers, the public transit passengers, and the pedestrians. We also assume that each public transit carries 20 passengers which is equivalent to 5 times that of automobiles (Aashtiani, 1979).

b) The public transit schedule is considered to be a transfer cost for pedestrians who change their mode from walking to public transit.

c) The problem is deterministic, so the transportation demands and supplies are known and there is no randomness in road capacity.

d) The demands for modes are not fixed and not known in advance, and the travelers decide on their mode of transportation based on their travel cost.

\subsection{Network Representation}

We propose a transportation network that consists of a network $\mathcal{G}(\mathcal{N}, \mathcal{L})$ made up of a set of links, $\mathcal{L}$, representing road segments, public transit lines and sidewalks and crosswalks, and a set of nodes, $\mathcal{N}$, representing intersections between these links. Network $\mathcal{G}(\mathcal{N}, \mathcal{L})$ is reconstructed from a given onlyautomobile network $G(N, L)$. The original network $G(N, L)$ is made up of a set of links, $L$, representing only road segments, and a set of nodes, $N$, representing the intersections between the road segments. The original and the reconstructed networks for the Small network instance are illustrated in Fig. 1. The original network $G(L, N)$ in this example as illustrated in Fig. 1(a) consists of $N=\{1,2,3,4\}$, representing nodes for the automobile mode, and $L=\{(1,3),(1,4),(2,1),(2,3),(3,4)\}$, representing road segments (Parsafard et al., 2015). 
The reconstruction mapping function $\Re$ is a process that takes the original network $G(N, L)$ and transforms it into a new network that, in addition to road segments and their intersections, also includes public transit lines and S\&C with separate links and nodes. For each link $l \in L$, representing a road segment in $G$, links $l_{s 1}$ and $l_{s 2}$ are added to the left and right hand sides of $l$ in parallel, to represent leftand right- hand sidewalks, respectively, for road segment $l$. Also, two crosswalk links, $l_{c_{\text {begin }}}$ and $l_{c_{\text {end }}}$, are added, one at the beginning and one at the end of each link $l$ to the network. We refer to these crosswalks as the begin- and end- crosswalks. The crosswalk links cross the automobile link $l$ and connect the left- and right- hand sidewalks. In addition to sidewalk and crosswalk nodes and links, public transit nodes and links are also added to the network. To connect these walking and public transit nodes and links to the original network, transfer links (representing the switch from one mode to another) are added to the network. For each origin and destination in the set of origin-destination pairs, dummy origin and destination nodes are added to the network. To ensure connectivity of the network, each dummy node is connected to its nearest sidewalk node using a connector link with zero travel time. This also ensures that travelers start and end their trips by walking (using sidewalk links). The reconstructed network for the Small network instance is illustrated in Fig. 1 (b). In this figure, the dummy nodes are shown in green and are represented by 5, 6, 7 and 8. Walking nodes are represented with three digit numbers, e.g. 231. The public transit nodes are represented with four digit numbers, e.g. 1001 (Parsafard et al., 2015).

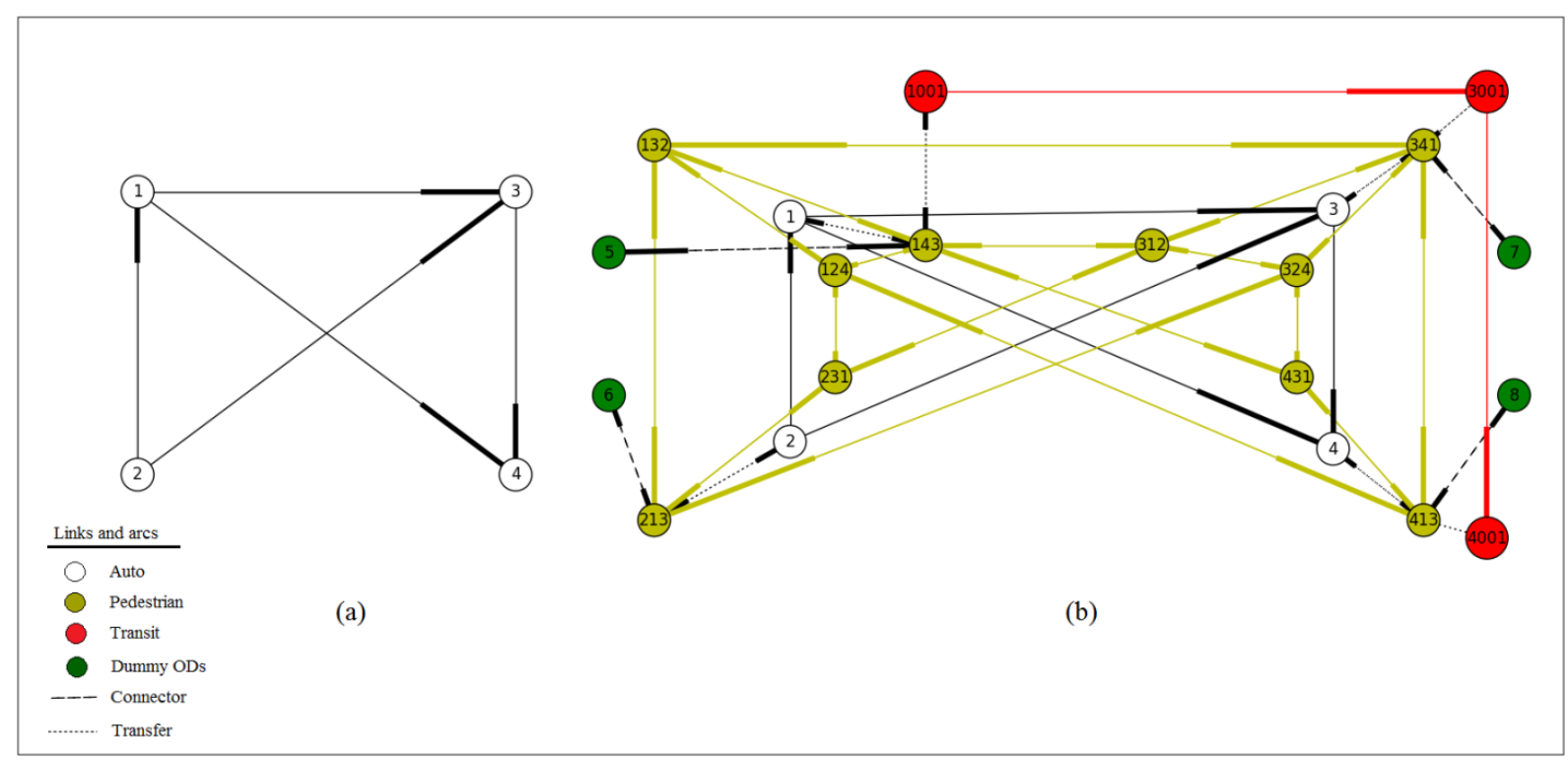

Fig. 1. Small network, (a) original network (b) reconstructed network. 


\subsection{Mixed integer bi-level programming formulation}

In the proposed bi-level model, the upper level problem locates and installs S\&C on the reconstructed network, and the lower level problem solves the corresponding traffic assignment problem and user equilibrium. The reconstructed network is represented with $\mathcal{G}(\mathcal{N}, \mathcal{L})$ in which $\mathcal{N}$ and $\mathcal{L}$ are the set of nodes and links. Links in $\mathcal{L}$ can be divided into six categories: $A$ for automobile links (which is equal to set $L$ in the original network $G(L, N)$ ), $T$ for public transit links, $S$ for sidewalk links, $C$ for crosswalk links, $F$ for transfer links that connect walking links to automobile and public transit links, and $R$ for connector links which connect the whole network together. The sets of incoming and outgoing links to and from node $i \in \mathcal{N}$, respectively, are represented with $I(i)$ and $O(i) . M$ is the set of transportation modes that contains indices ' $a$ ', ' $t$ ' and ' $w$ ' representing automobile, public transit and walking modes, respectively. $K$ represents the set of trips in the transportation network. For trip $k \in K, d_{k}$ is the transportation demand, starting from the origin $\mathcal{O}_{k}$ and ending at the destination $\mathcal{D}_{k}$. The set of traffic calming facilities (S\&C) is represented with $J$. Although we assume that the installation of sidewalks ("s") includes implementing both left- and right- hand sidewalks, for the begin- and end- crosswalks (" $c_{\text {begin }}$ " and " $c_{\text {end }}$ "), we assume they can be installed independently. If the set of traffic calming facilities available for link $l_{a} \in A$ (the subscript $a$ indicates that link $l$ is an automobile link) is represented by $J_{l_{a}}$, then the decision for city planners to make is whether to install the traffic calming facility $j \in J_{l_{a}}$ that costs $c_{J_{l_{a}}}$ on the link $l_{a}$ and imposes the transportation cost $\varphi_{\mathrm{l}}($.$) . The criterion for$ this decision making is whether the overall transportation cost decreases while pedestrians' safety increases. This decision is limited to budget constraint on implementing traffic calming facilities (not exceeding $b$ ) on the transportation network. Table 1 shows all the notations used in the proposed model.

As mentioned earlier, two traffic calming facilities are considered in this research, sidewalks and crosswalks. For crosswalks, we assume that there can be two crosswalk lines on each automobile link: one at the beginning (represented by $l_{c_{\text {begin }}}$ ) and one at the end (represented by $l_{c_{\text {end }}}$ ). We assume that the begin- and end-crosswalks can exist and work independently. However, for sidewalks, we assume that the left- and the right-hand sidewalks are dependent and cannot exist independently, meaning that if we build a sidewalk for a road, we build it for both sides of the road.

We formulate OTCIMTP as a link-based model. Each link in the transportation network has a transportation cost that includes the travel time converted to a dollar value. 
Table 1. Notations

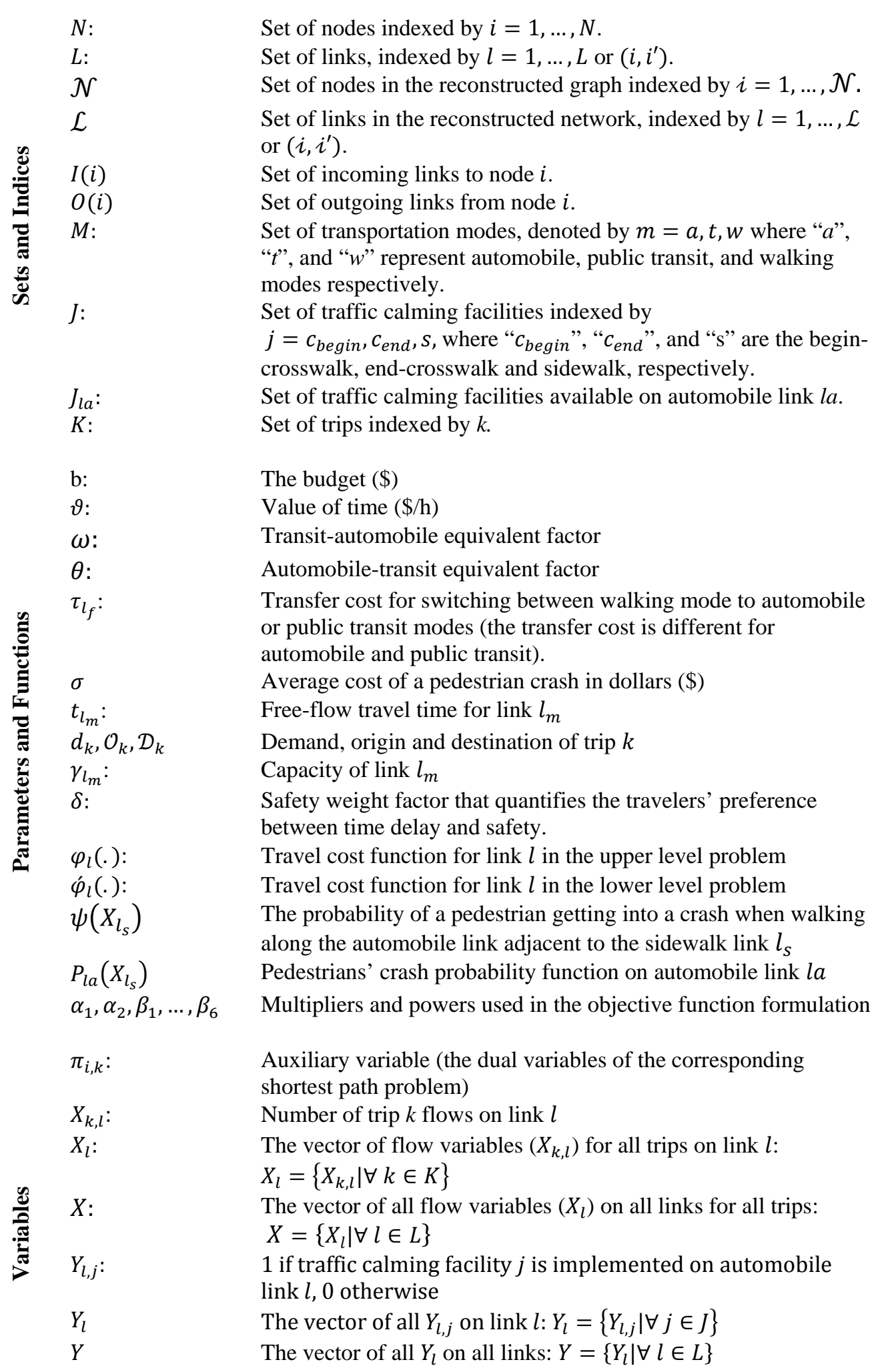




\subsubsection{Travel cost function}

For automobile links, $l_{a} \in A$, the travel cost function is as follows:

$$
\begin{aligned}
& \varphi_{l_{a}}(X, Y)=\left(t_{l_{a}}\left(1+\alpha_{1}\left(\frac{\sum_{k \in K} X_{k, l_{a}}+\sum_{k \in K} \omega X_{k, l_{t}}}{\gamma_{l_{a}}}\right)^{\beta_{1}}\right)\right. \\
& +\left(\left(\frac{\sum_{k \in K} X_{k, l_{s 1}}}{\gamma_{l_{s 1}}}\right)^{\beta_{2}}+\left(\frac{\sum_{k \in K} X_{k, l_{s 2}}}{\gamma_{l_{s 2}}}\right)^{\beta_{2}}\right) \times\left(1-y_{l_{a}, s 1}\right) \\
& \left.+\left(\frac{\sum_{k \in K} X_{k, l_{c_{\text {begin }}}}}{\gamma_{l_{c_{\text {begin }}}}}\right)^{\beta_{3}} \times\left(y_{l_{a, c_{\text {begin }}}}\right)+\left(\frac{\sum_{k \in K} X_{k, l_{c_{\text {end }}}}}{\gamma_{l_{c_{\text {end }}}}}\right)^{\beta_{3}} \times\left(y_{l_{a, c_{\text {end }}}}\right)\right) \times \vartheta+\mu_{l_{a}}
\end{aligned}
$$

The travel cost of using the automobile mode on link $l_{a} \in A$ is affected by ( $a$ ) the amount of flow on automobile link $l_{a}$, $(b)$ the amount of flow on the other links associated with link $l_{a}$ (public transit and walking modes), and $(c)$ whether traffic calming facilities, S\&C, are installed on link $l_{a}$. To incorporate these three factors into the travel cost of automobile links, we divide the automobile travel cost into three parts. We use the coefficient $\vartheta$ (the value of time) to convert all these travel costs to a dollar value.

Part $(i)$ reflects the effect of traffic flows of the automobile mode in conjunction with the flows of public transit on link $l_{a}$. In this formula, $t_{l_{a}}$ is the free flow travel time on link $l_{a}$. The quantities $\alpha_{1}$ and $\beta_{1}$ are model parameters. $X_{k, l_{a}}$ and $X_{k, l_{t}}$ are the amount of flows for automobile and public transit respectively. $\gamma_{l_{a}}$ is the capacity of link $l_{a}$. Since there is a difference between the size of automobile and public transit options (say, the capacity of the public transit is $\omega$ times bigger than that of automobile), we add $\omega$ as a multiplier to $X_{k, l_{t}}$ to make up for this difference. We refer to $\omega$ as the transit-automobile equivalent factor.

Part (ii) reflects the effect of traffic flows on the left- and right-hand sidewalks on automobile link $l_{\mathrm{a}}$. $X_{k, l_{s} 1}$ and $X_{k, l_{s} 2}$ are the number of flows in the left- and right-hand sidewalks, respectively, and $\gamma_{\mathrm{l}_{\mathrm{s} 1}}$ and $\gamma_{l_{\mathbf{s} 2}}$ are the capacity of the left- and right hand sidewalks respectively. The quantity $\beta_{2}$ is a model parameter. When a sidewalk is installed, we assume that both the left- and the right-hand sidewalks are built on the sides of a street. $\mathrm{y}_{\mathrm{l}_{\mathrm{a}, \mathrm{s}}}$ indicates whether or not sidewalks are installed on the street represented by link $\mathrm{l}_{\mathrm{a}}$. When $\mathrm{y}_{\mathrm{a}, \mathrm{s1}}=1$, it indicates that two sidewalks are installed on both sides of $l_{a}$. Installing sidewalks on a link creates separate walkways for pedestrians which can decrease the travel cost of the automobile mode, as the flow of pedestrians no longer interferes with the flow of automobiles.

Part (iii) reflects the effect of traffic flows on crosswalks (both the begin- and end-crosswalks) on automobile link $\mathrm{l}_{\mathrm{a}}$. In this formula $\mathrm{X}_{\mathrm{k}, \mathrm{l}_{\mathrm{c}_{\text {begin }}}}$ and $\mathrm{X}_{\mathrm{k}, \mathrm{l}_{\mathrm{c}} \text { end }}$ are the amount of flows in the begin- and end 
crosswalks respectively, and $\gamma_{\mathrm{l}_{\mathrm{c}_{\text {begin }}}}$ and $\gamma_{\mathrm{l}_{\mathrm{end}}}$ are the capacity of these crosswalks. The quantity $\beta_{3}$ is a model parameter. $y_{l_{a, c_{b e g i n}}}$ and $y_{l_{a, c_{e n d}}}$ are decision variables indicating whether or not to install sidewalks (at the beginning or at the end of a street). We assume the begin- and end-crosswalks can be implemented separately and independently (as opposed to sidewalks). Installing begin- or end-crosswalks can encourage more pedestrians to cross the automobile link $l_{a}$ and increases its travel cost. This is reflected in part (ii) of equation (1). When a crosswalk is installed $\left(y_{l_{a, c_{b e g i n}}}\right.$ or $\left.y_{l_{a, c_{e n d}}}=1\right)$, the flow of pedestrians affects the travel cost of automobile on link $l$. In this formula, $\mu_{l a}$ represents the out-of-pocket cost (the indirect costs) that automobile drivers have to pay (such as gas, insurance etc.) for using automobile (there is no such cost when walking or using public transit).

The travel cost function for public transit link $\varphi_{l_{t}}($.$) is as follows:$

$\varphi_{l_{t}}(X, Y)=\vartheta \times t_{l_{t}}\left(1+\alpha_{2}\left(\frac{\sum_{k \epsilon K}\left(X_{k, l_{t}}+\theta X_{k l_{a}}\right)}{\gamma_{l_{t}}}\right)^{\beta_{4}}\right)$

The travel cost of public transit reflects the effect of traffic flows of public transit in conjunction with the flows of automobile on link $l_{t}$. In this formula, $t_{l_{t}}$ is the free flow travel time on link $l_{t}$. The quantities $\alpha_{2}$ and $\beta_{4}$ are model parameters, and $\gamma_{l_{t}}$ is the capacity of link $l_{t}$. Similar to how we treated the travel cost function (1), part ( $i$ ), we use a multiplier, $\theta$, this time for $X_{k, l_{a}}$, to make up for the difference between the capacity of an automobile and that of a public transit. We refer to this multiplier as the automobile-transit equivalent factor. We use the coefficient $\vartheta$ (the value of time) to convert the travel cost to a dollar value.

The travel cost function for crosswalk link $\varphi_{l_{c}}($.$) is as follows:$

$\varphi_{l_{c}}(X, Y)=\vartheta \times t_{l_{c}}\left(1+\propto_{3}\left(\frac{X_{l_{c}}}{\gamma_{l_{c}}}\right)^{\beta_{5}}\right)$

It reflects the effect of traffic flow of the crosswalk on link $l_{c}$. In this formula, $t_{l_{c}}$ is the free flow travel time for crossing a street on link $l_{c}$. The free flow travel time can be found based on the length of $l_{c}$ and walking speed. $X_{l_{c}}$ is the flow of pedestrians crossing an street using the crossover link $l_{c}$, which has the capacity of $\gamma_{l_{c}}$. The quantities $\alpha_{3}$ and $\beta_{5}$ are model parameters. We use the coefficient $\vartheta$ (the value of time) to convert the travel cost to a dollar value.

The travel cost function for sidewalk links $\varphi_{l_{s}}($.$) is as follows:$

$\varphi_{l_{s}}(X, Y)=\vartheta \times t_{l_{s}}\left(1+\propto_{4}\left(\frac{X_{l_{s}}}{\gamma_{l_{s}}}\right)^{\beta_{6}}\right)$ 


$$
+\left(1-y_{l_{s}}\right) \times \sigma P_{l_{a}}\left(X_{l_{a}}\right) X_{l_{s}}
$$

This travel cost function consists of two parts: part $(i)$ reflects sidewalks' travel time, and part (ii) reflects pedestrians' safety on sidewalks. Since travel time is in units of time unit and safety is in units of currency, we convert the travel time part to a dollar value using the multiplier $\vartheta$.

Part $(i)$ is a function of the effect of the flow of pedestrians $\left(X_{l_{s}}\right)$ on sidewalk link $l_{s}$. In this formula, $t_{l_{s}}$ is the free-flow travel time, and $\gamma_{l_{s}}$ is the capacity of link $l_{s}$. The quantities $\alpha_{4}$ and $\beta_{6}$ are model parameters.

Part ( $i i$ ) is the safety part and computes the expected cost of pedestrians' crashes (as a penalty cost in the absence of installed sidewalks) on the automobile link $l_{a}$ adjacent to sidewalk $l_{s}$. When no sidewalk is installed, pedestrians have to walk along the streets, which is unsafe. To compute the expected cost of pedestrians' crashes, we multiply the probability that a given pedestrian will get in a crash $\left(P_{l_{a}}\left(X_{l_{a}}\right) X_{l_{s}}\right)$ by the average cost of a pedestrian crash $(\sigma)$ (Gårder, 2004). The term $P_{l_{a}}\left(X_{l_{a}}\right)$ is the pedestrian crash probability function when pedestrians walk along the automobile link $l_{a}$. This function is obtained by dividing the total number of crashes on a given road segment by the total traffic flow on that segment. Then, by using a simple linear regression among all the streets, we calculate the crash probability function. For this regression we used the historical crash data from Starkville, Mississippi, U.S.A. (see [33] for more details).

In this formula, $y_{l_{s}}$ indicates whether or not a sidewalk is installed, which can enforce the cost associated with pedestrians' crashes in the absence of an installed sidewalk.

For sidewalk links, the travel cost function for the upper level problem (equation (4)) is slightly different from the travel cost function in the lower level. The travel cost function for the lower level is as follows:

$$
\begin{gathered}
\dot{\varphi}_{l_{s}}(X, Y)=\quad \\
\quad(1-\delta) \times t_{l_{s}}\left(1+\propto_{4}\left(\frac{X_{l_{s}}}{\gamma_{l_{s}}}\right)^{\beta_{6}}\right) \\
+\delta\left(1-y_{l_{s}}\right) \times \sigma \psi\left(X_{l_{s}}\right)
\end{gathered}
$$

The travel cost function for the lower level also consists of two parts: part ( $i$ ) reflects sidewalks' travel time, and part ( $i i$ ) reflects pedestrians' safety on sidewalks. Part $(i)$ is similar to what is used in equation (4). However, here we also use an adjustment safety weight factor, $\delta$, to combine the travel time part and the safety part. The adjustment weight factor can be used by city planners for find a balance between travel safety and travel time when designing a transportation network. Part (ii) computes the expected cost for an individual when walking along the automobile link $\mathrm{l}_{\mathrm{a}}$ (adjacent to the sidewalk $\mathrm{l}_{\mathrm{s}}$ ) for which the sidewalk has not been built. In this formula, $\psi\left(\mathrm{X}_{\mathrm{l}_{\mathrm{s}}}\right)$ is the probability that a pedestrian gets into a 
crash when walking along the automobile link $\mathrm{l}_{\mathrm{a}}$ (due to the lack of an installed sidewalk) and is computed as follows:

$\psi\left(X_{l_{s}}\right)=\left(\frac{P_{l_{a}}\left(X_{l_{a}}\right) X_{l_{s}}}{0.01 \times \gamma_{l_{s}}}\right)$

The numerator in (5a) computes the expected number of pedestrians' crashes on automobile link $l_{a}$ when pedestrians walk along the street. The denominator in (5a) is an estimate of the average number of pedestrians walking along automobile link $l_{a}$. Due to a lack of data, we assume the average number of pedestrians walking along a street is $1 \%$ of the capacity of the adjacent sidewalk if it was built.

The links that connect the pedestrian links to automobile and public transit links are called "transfer links.” The travel cost function for transfer links $\varphi_{l_{f}}($.$) is a constant value which indicates the total$ walking time to reach the transfer station and the total waiting time in the transfer station:

$\varphi_{l_{f}}(X, Y)=\vartheta \times \tau_{l_{f}}$

There are two types of transfer links: automobile-walking and transit-walking transfer links. In order to transfer from public transit to automobile (or automobile to public transit), both of these transfer links must be used.

Finally, the travel cost function for connector links $\varphi_{l_{r}}($.$) is assumed to be 0$ because these links are hypothetical:

$\varphi_{l_{r}}(X, Y)=0$

The objective functions in the upper level and the lower level are different. This is due to incorporating pedestrians' safety on sidewalks into the objective function of the transportation problem in the upper level problem. However, pedestrians' safety is also incorporated into the objective function of the lower level problem. We assume that the travel cost for all links, except sidewalk links, is the same for the upper level problem and the lower level problem: $\dot{\varphi}_{l}(X, Y)=\varphi_{l}(X, Y) \quad \forall l \epsilon \mathcal{L} \backslash\left\{l_{s} \mid s \in S\right\}$.

\subsubsection{Mathematical formulation for OTCIMPT:}

The proposed mathematical model is formulated as follows:

$\operatorname{Min} \sum_{k \in K} \sum_{l \in L} \varphi_{l}(X, Y) X_{k, l}$

s.t. 


$$
\begin{array}{cl}
X_{k, l}\left(\dot{\varphi}_{l}(X, Y)-\left(\pi_{i^{\prime}, k}-\pi_{i, k}\right)\right)=0 & \forall l=\left(i, i^{\prime}\right) \in \mathcal{L}, k \in K \\
\sum_{l \in O\left(\mathcal{O}_{k}\right)} X_{k, l}=d_{k} & \forall k \in K \\
\sum_{l \in I(i)} X_{k, l}-\sum_{l^{\prime} \in O(i)} X_{k, l^{\prime}}=0 & \forall i \in \mathcal{N} \backslash\left\{\mathcal{O}_{k}, \mathcal{D}_{k}\right\}, k \in K \\
\sum_{j \in J} \sum_{l \in L} c_{j, l} y_{l, j} \leq b & \\
X_{k, l} \geq 0 & \\
\pi_{i k} \geq 0 & \forall k \in K, l \in \mathcal{L} \\
y_{l, j} \in\{0,1\} & \forall k \in K, i \in I \\
& \forall j \epsilon J, l \in L
\end{array}
$$

The objective function (8) is to minimize the total transportation cost, including the cost of travel time due to a lack of pedestrians' safety, in the network (in the objective function, a lack of safety is penalized with a dollar-value equivalent; therefore, by minimizing total cost, we are also minimizing lack of safety). Constraints (9) enforce the optimal flow solution to be at travel cost equilibrium. Constraints (10) require that all of the demand flows through the network for every trip. Constraints (11) enforce conservation of flow for all nodes in the network. Constraint (12) is the budget constraint, and constraints (13) to (15) serve to restrict the range of variables.

Though the problem as a whole is a non-convex mixed-integer nonlinear programming problem, the following proposition shows that the lower level problem is convex.

Proposition 1: The objective function of the lower level problem, $\varphi_{l}($.$) , is convex.$

Proof: See Appendix.

For any solution to the upper level problem which locates and installs S\&C, we use the nonlinear complementary algorithm (Aashtiani, 1979) to solve the traffic assignment and user equilibrium in the lower level. The nonlinear complementary algorithm does not require the lower level problem to be convex. However, since the problem is convex, any other algorithm that requires convexity can also be used. 


\section{Methodology}

Solving a bi-level network design problem is difficult as the problem is NP-hard. In fact, as BenAyed and Blair (1990) showed, even a linear bi-level problem (or bi-level linear problem; BLP) is NPhard. Therefore, solving the problem for large scale instances using exact solution methods requires extensive computational resources. Studies on multimodal network design problems are very few, and the solution techniques used in these studies are mostly approximate methods (Farahani et al., 2013). A single level formulation for the problem is more tractable (Farvaresh and Sepehri, 2011) but not always possible. Thus, heuristic algorithms are often developed for transportation network design problems (Farahani et al., 2013). Meta-heuristic algorithms are also very common in transportation network design problems, especially for large scale problems (Drezner and Salhi, 2002; Miandoabchi et al., 2011a, 2011b; Yamada and Febri, 2015; Yang et al., 2007). A major benefit of these methods is that they run much faster than exact methods. Some common methods of this kind include Genetic Algorithm, Simulated Annealing, Tabu Search, and Ant Colony Optimization (Farahani et al., 2013).

To solve the proposed bi-level transportation network design problem in this paper, we use an exact approach and two heuristic ones. For the exact approach we implement the model in YALMIP (version 20141030) (Lofberg, 2004) and solve it using the BARON solver (version v1.69) (Sahinidis, 2014), a computational system that can solve mixed integer nonlinear programming problems. We also develop a customized greedy heuristic (GH) and a simulated annealing (SA) algorithm. These algorithms are used to solve the upper level problem that is where to install S\&C in the network considering the limited budget. Then, a nonlinear complementary algorithm (Aashtiani, 1979) is used to solve the lower level problem (the user equilibrium traffic assignment problem on the reconstructed network). We use a linklist dynamic data structure proposed by Toobaei et al. (2010), which was reported to outperform the Frank-Wolfe algorithm (Frank and Wolfe, 1956). The advantages of the nonlinear complementary algorithm are its speed and the fact that it allows for a general cost function (i.e. the travel cost is a function of all the flows in the network). In the following sections, we describe in more detail the customized greedy heuristic and the simulated annealing algorithm proposed for this problem.

\subsection{Greedy Heuristic}

The greedy heuristic (GH) is a simple heuristic algorithm that makes the locally optimal choice at each stage with the hope of finding a global optimum. The GH in this study starts with a null solution $X$ (no sidewalks or crosswalks installed) and iteratively finds a new solution suggesting where to install a new sidewalk or crosswalk from the set of all possible options $(X \dot{X})$ until the budget is exhausted. For a given solution $X$, the nonlinear complementary algorithm is used to compute its corresponding objective 
value, $f(X)$. The benefit-cost ratio $\frac{f(X)}{\operatorname{Cost}(X)}$ is computed to evaluate the suitability of that solution. The solution that has the largest benefit-cost ratio in that iteration is accepted (i.e. the corresponding sidewalk or crosswalk is installed). Additional S\&C are installed at successive iterations in a similar fashion until the budget is exhausted.

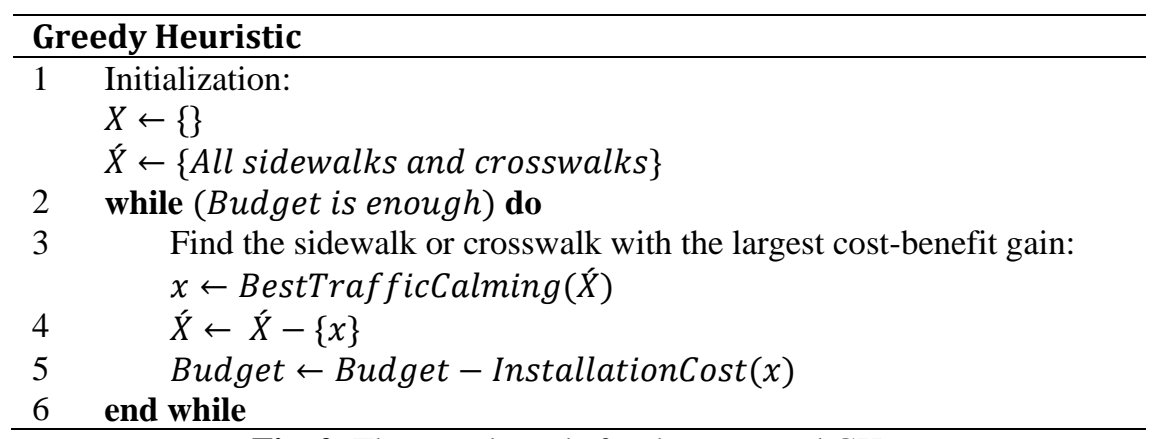

Fig. 2. The pseudo code for the proposed GH

In this study, we assume that the cost of installing S\&C at any location is the same. Therefore, instead of $\frac{f(X)}{\operatorname{Cost}(X)}$ we can use $f(X)$ in evaluating solution $X$. The pseudo code of the GH is shown in Fig. 2.

\subsection{Simulated Annealing}

Simulated annealing (SA) is a probabilistic metaheuristic that emulates the physical gradual cooling process that produces high quality crystals. The method was proposed by Kirkpatrick and Vecchi (1983). Variants of SA algorithms have been successfully applied to different optimization problems (Aliakbarian et al., 2015; Kia et al., 2012; Madadi et al., 2014; Miandoabchi et al., 2013).

An SA algorithm repeats an iterative neighbor generation procedure and follows search directions that improve the objective function value. To escape from local optima, the SA algorithm offers the possibility to accept worse solutions with a probability that decreases as the algorithm moves toward completion. In each iteration, the difference between the objective value of the current solution $f(X)$ and the new solution $f(\dot{X})$ is evaluated as $\Delta=f(X)-f(\dot{X})$. If $\Delta \geq 0$ (for a minimization problem), the new solution $\dot{x}$ is accepted; otherwise, it will be accepted with a probability of $p=\exp \left(\frac{\Delta}{T}\right)$, in which $T$ is a parameter called the temperature of the current state. The factors that influence acceptance probability are the degree of objective function value degradation $\Delta$ and the temperature $T$. Smaller degradation and higher temperature induce higher acceptance probability. The temperature can be controlled by a process called the cooling schedule, which specifies how it should be progressively reduced to make the procedure more selective as the search progresses to neighborhoods of good solutions (Bouleimen and Lecocq, 2003). 
The cooling schedule starts with a high temperature $T_{\max }$ so that it allows acceptance of new neighbor solutions with higher probability. An attenuation factor $\propto(0<\propto<1)$ is used to decrease the temperature in each iteration, so the acceptance probability decreases. The algorithm is terminated when the current temperature reaches the minimum temperature $\left(T_{\min }\right)$.

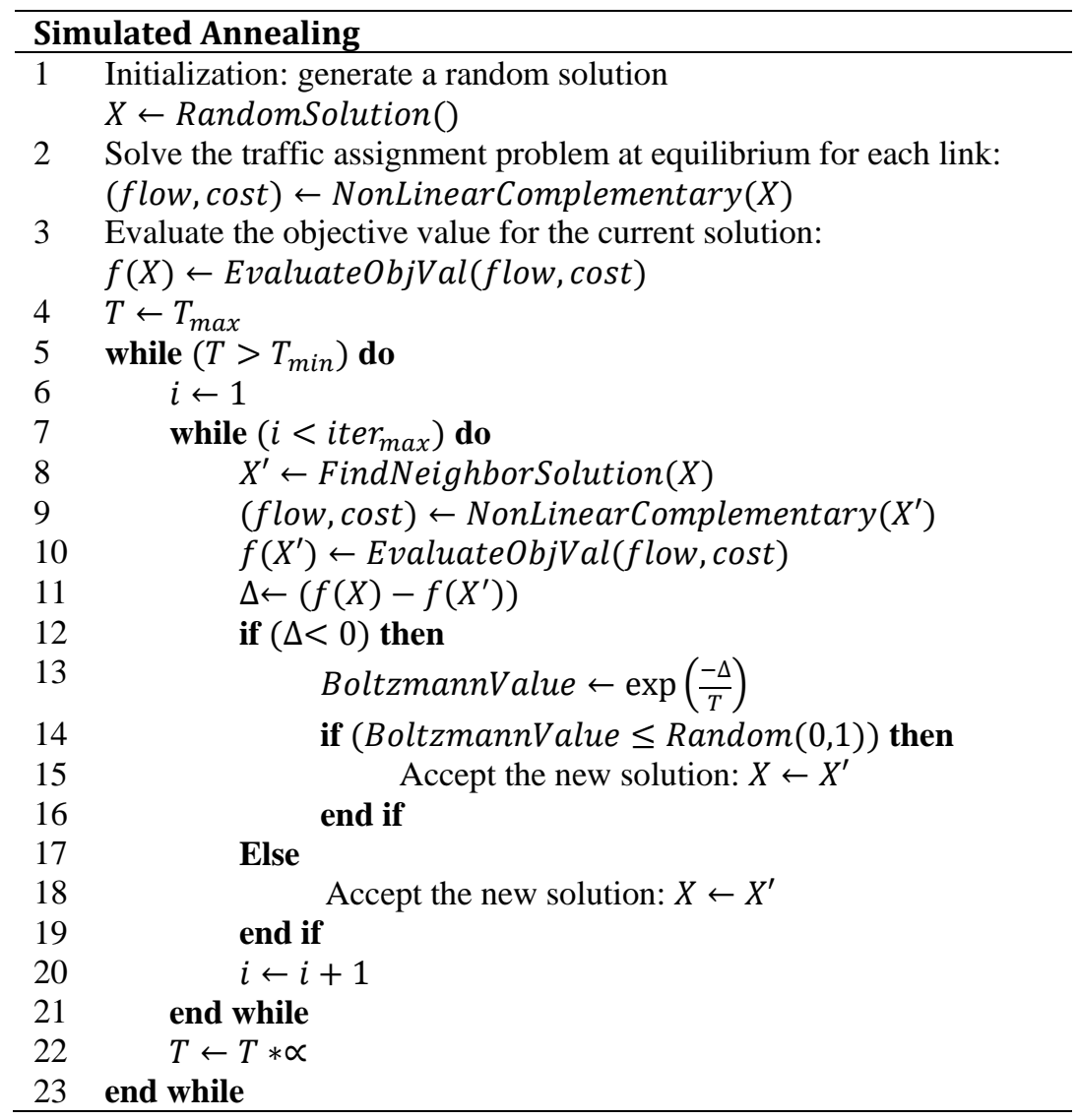

Fig. 3. The pseudo code for the proposed SA

Table 2. The SA parameters and their values

\begin{tabular}{cc}
\hline Parameter & Value \\
\hline$T_{\max }$ & 42000 \\
$T_{\min }$ & 0.00001 \\
Iter & 20 \\
$\propto$ & 0.80 \\
\hline
\end{tabular}

The neighborhood search used in in this paper consists of two steps: (1) removing an installed traffic calming facility and (2) installing a traffic calming facility. For a given solution, we first select an automobile link arbitrarily from the pool of automobile links with installed traffic calmings and uninstall its traffic calming (if more than one traffic calming is installed on that automobile link, one 
is arbitrarily selected and uninstalled). Then, we update the budget (the cost of the traffic calming that was uninstalled is reimbursed). Next, we arbitrarily select another automobile link (from the pool of all automobile links) and install a traffic calming (either sidewalk or crosswalk, arbitrarily chosen) on that automobile link. If the selected automobile link already has that traffic calming installed, we discard that link and select another one. Fig. 3 shows the pseudo code for the proposed SA, and Table 2 shows the tuned parameters used in the cooling schedule.

\section{Numerical Experiments}

Three sample networks are used for experimentation: a small hypothetical network (called "Small network”), the Hearn network (Hearn and Ramana, 1998) and the Sioux Falls network. The characteristics of these networks are given in Table 3. For the Sioux Falls network, the demand for each origin-destination pair is available. However, for the Small and Hearn networks, which are hypothetical transportation network instances, no such data are available. Therefore, we generate these data arbitrarily for experimentation. Table 4 shows the arbitrarily-generated demand data that we use in this paper. Table 5 shows the parameters we use in the travel cost functions that were presented in section 2.

Table 3. Sample Transportation Networks

\begin{tabular}{|c|c|c|c|c|c|}
\hline \multirow[b]{2}{*}{ Network } & \multirow[b]{2}{*}{$\begin{array}{l}\text { Num. of OD } \\
\text { pairs }\end{array}$} & \multicolumn{2}{|c|}{ Original network } & \multicolumn{2}{|c|}{ Reconstructed Network } \\
\hline & & $\begin{array}{c}\text { Num. of } \\
\text { nodes }\end{array}$ & $\begin{array}{c}\text { Num. of } \\
\text { links }\end{array}$ & $\begin{array}{c}\text { Num. of } \\
\text { nodes }\end{array}$ & Num. of links \\
\hline Small & 4 & 4 & 5 & 21 & 65 \\
\hline Hearn & 4 & 9 & 18 & 55 & 192 \\
\hline Sioux Falls & 552 & 24 & 76 & 143 & 523 \\
\hline
\end{tabular}

Table 4. Demand for different OD pairs for the Small and Hearn Networks

\begin{tabular}{cccccc}
\hline \multicolumn{3}{c}{ Small Network } & \multicolumn{3}{c}{ Hearn Network } \\
OD Pairs & Demand & OD Pairs & Demand \\
from & to & & from & to & \\
\hline 5 & 7 & 10 & 10 & 12 & 20 \\
5 & 8 & 40 & 10 & 13 & 40 \\
6 & 7 & 20 & 11 & 12 & 60 \\
6 & 8 & 60 & 11 & 13 & 80 \\
\hline
\end{tabular}

The parameters used as coefficients in the travel cost functions in Table 5 are commonly used values. It is assumed that a public transit carries, on average, 20 passengers; 5 times more than that of an automobile (Aashtiani, 1979). This makes $\omega=\frac{4}{20}=0.2$, and $\theta=\frac{20}{4}=5$ as shown in Table 5. The average cost of a pedestrian crash $(\sigma=\$ 78300)$ is computed using data from Gårder (2004). The 
Highway Economic Recruitments System (HERS) (Vandervalk et al., 2014) considers $\$ 19.86$ as the value of time (per hour), which is equal to $\$ 0.33$ per minute. Therefore we assume that $\vartheta=0.33$ (the value of time). The coefficients of the intercept and the slope of the linear regression model $\left(P_{l a}\left(X_{l a}\right)\right)$ that is used in the pedestrian crash probability function in presented equations (4) and (5a) are $1.7^{-7}$ and $3.59^{-10}$ respectively. Therefore, $P_{l a}\left(X_{l a}\right)=1.7^{-7} X_{l s}+3.59^{-10}$ (for more detail we refer the reader to Parsafard et al. (2015)).

Table 5. Parameters' values in travel cost functions used in this study

\begin{tabular}{ccc}
\hline Parameter & Value & Description \\
\hline$\alpha_{1}$ & 0.15 & Coefficient of the BPR-like function in (1) \\
$\alpha_{2}$ & 0.15 & Coefficient of the BPR-like function in (2) \\
$\alpha_{3}$ & 2 & Coefficient of the BPR-like function in (3) \\
$\alpha_{4}$ & 2 & Coefficient of the BPR like function in (4) and (5) \\
$\beta_{1}$ & 4 & Power in equation (1) \\
$\beta_{2}, \beta_{3}$ & 2 & Power in equation (1) \\
$\beta_{4}$ & 4 & Power in equation (2) \\
$\beta_{5}$ & 2 & Power in equation (3) \\
$\beta_{6}$ & 2 & Power in equation (4) and (5) \\
$\omega$ & 0.2 & Public transit to automobile equivalent factor in \\
& 5 & equation (1) \\
$\theta$ & 5 & The cost for transferring from the walking mode to \\
& & automobile and public transit modes respectively and \\
$\tau$ & 3,5 & vice versa (6) \\
$\sigma$ & 78300 & Average cost of a pedestrian crash in dollars (4), (5) \\
$\vartheta$ & 0.33 & Value of time in (1)-(6)
\end{tabular}

\subsection{Computational comparison}

We used the BARON solver to solve the mathematical model. After running for 24 hours, BARON did not provide any feasible solution, even for the Small network. On the other hand, the GH and the SA produced competitive solutions in a reasonable time (as shown in Table 5). Table 5 shows the computational results and the solutions produced by the SA and the GH algorithms after installing S\&C in the transportation networks. As can be seen in the table, the two algorithms produced solutions of similar quality. However, the SA performs lightly better for the larger Sioux Falls network. The SA is also much faster for the Sioux Falls network; however, it is slower for the Small and Hearn networks. As shown in Table 5, the results for all three networks suggest that carefully installing S\&C reduces the 
overall transportation cost; however, the cost reduction is more significant in the Sioux Falls network, with a 59\% decrease in the overall cost (according to the solution produced by the SA).

Table 5. A comparison of the computational performance of the SA and the GH (\% reduction in cost $=\frac{f\left(x^{0}\right)-f\left(x^{*}\right)}{f\left(x^{0}\right)}, x^{0}$ : The null solution i.e. when no $S \& C$ are installed, $\mathrm{x}^{*}$ : Solution found by our algorithms).

\begin{tabular}{ccccc}
\hline \multirow{2}{*}{ Networks } & \multicolumn{2}{c}{ \% Reduction in cost } & Time (sec) \\
& SA & GH & SA & GH \\
\hline Small & $\% 12$ & $\% 12$ & 20.7 & 0.92 \\
Hearn & $\% 5$ & $\% 5$ & 31 & 2.6 \\
Sioux Falls & $\% 59$ & $\% 58$ & 199 & 504 \\
\hline
\end{tabular}

The average demand per OD pair for the Small, Hearn and the Sioux Falls networks is 33, 50, and 653 , respectively, and the average demand per link is 2, 1 and 689, respectively.

We speculate that the higher demand per link and per OD pair in the Sioux Falls network might be the reason for the higher reduction in cost for this network. We will investigate the impact of demand for these networks later in section 4.3. Regarding the computation time of the two algorithms for the hypothetical Small and Hearn networks, as shown in Table 5, the GH is faster than the SA; however, for the Sioux Falls network, the SA is faster than the GH. To investigate the cause of these differences, we counted the number of times that the nonlinear complementary algorithm is called by these two algorithms (for solving the user equilibrium in the lower level problem). We learned that for the Small and Hearn networks, the nonlinear complementary algorithm is called more often in the SA than the GH. For the Sioux Falls network, however, as the budget increases, the nonlinear complementary algorithm is called more often in the GH than the SA. Therefore, the GH becomes computationally more expensive and less efficient than the SA for the larger Sioux Falls network (Fig. 4). In summary, for the larger transportation network (the Sioux Falls), the SA outperforms the GH both in solution quality and computation time.

As shown in Table 5, installing S\&C reduces the total transportation cost. To test the robustness of these results, we do a sensitivity analysis. We study the impact of S\&C on the total transportation cost under different conditions such as having a different budget for installing S\&C, being subject to different demand in the transportation networks, and using different values for the safety weight factor. 


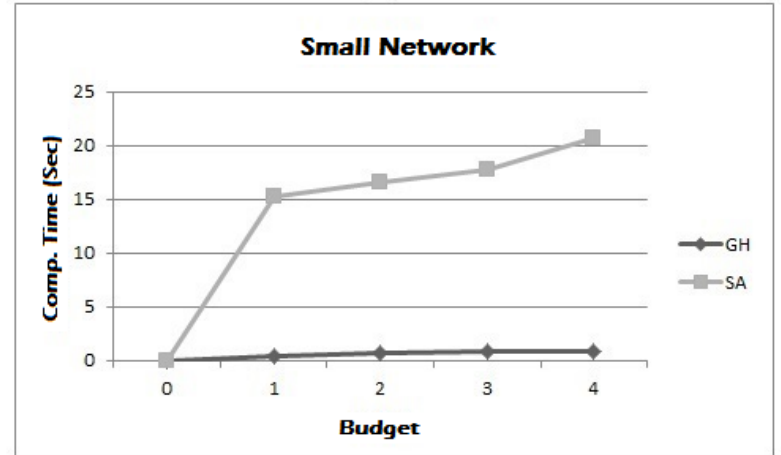

(a)

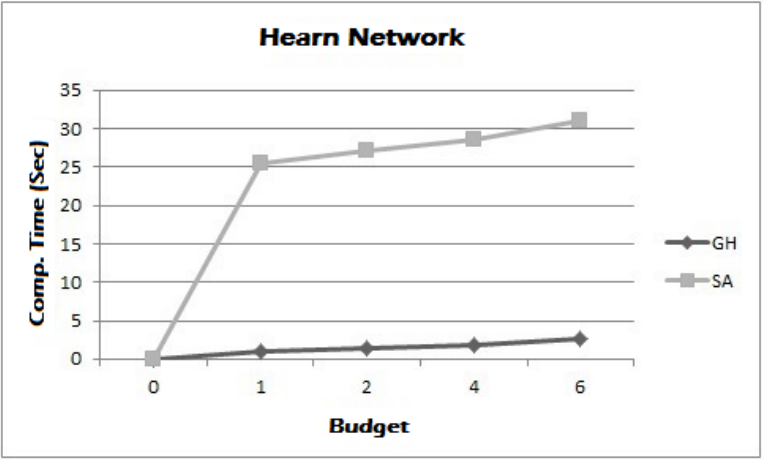

(b)

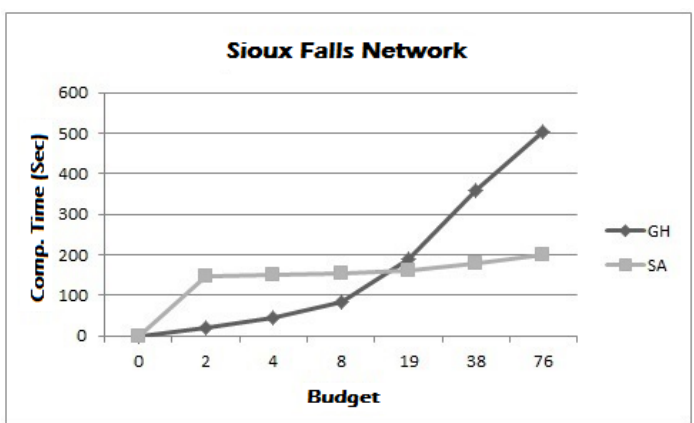

(c)

Fig.4. Computation time, the GH vs. the SA for the

(a) Small network, (b) Hearn network and (c) Sioux Falls network

\subsection{Sensitivity analysis: Budget}

In the previous experiment (Table 5), we assumed that the budget for installing S\&C was unlimited. To test the performance of the GH and the SA with a limited budget, we ran another set of experiments imposing a limit on the budget for installing S\&C. Our results show that, as the budget increases and more S\&C are installed, the overall transportation cost decreases in all three networks; however, for the Sioux Falls network, this change is more significant. For all three networks, as budget increases, the degree by which the overall cost decreases diminishes, and there exists a point where adding more S\&C no longer affects the overall cost (see Fig. 5.) 


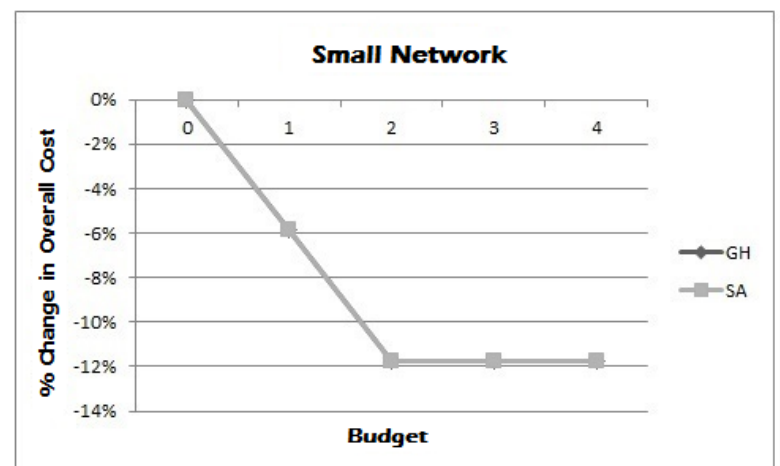

(a)

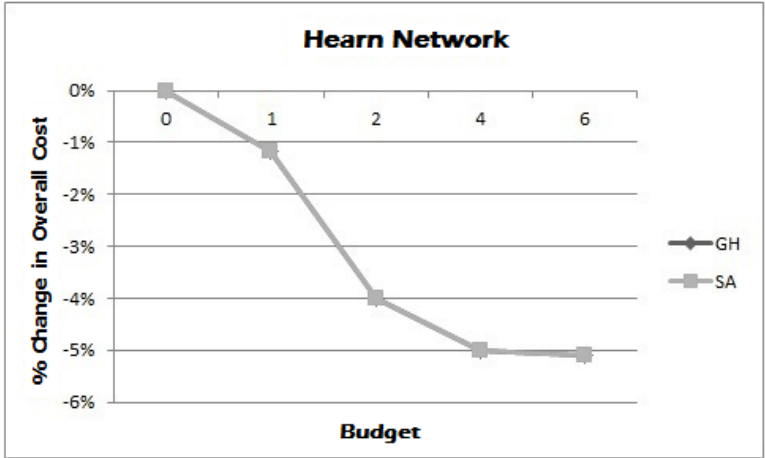

(b)

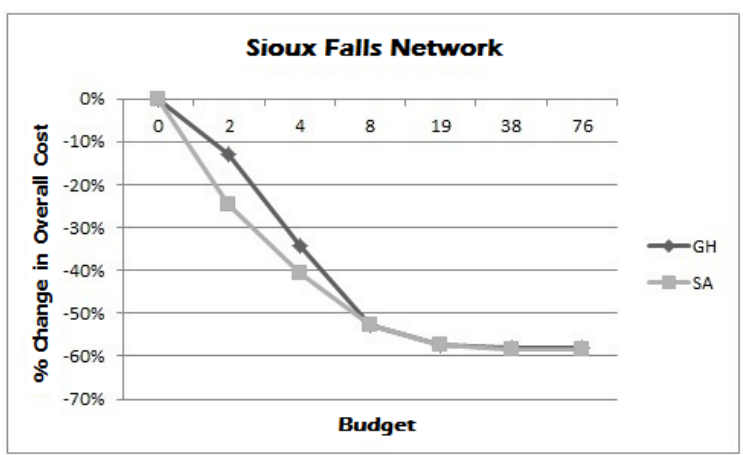

(c)

Fig.5. Percent change in the overall cost for different budget values:

(a) Small Network, (b) Hearn Network, and (c) Sioux Falls Network

The reason that such a point exists is that, after a certain number of S\&C are installed and separate walkways are available for pedestrians to use, the pedestrians no longer walk along the streets and interfere with automobile and public transit traffic. Accordingly, adding more S\&C can no longer reduce the transportation cost. Therefore, it is not recommended to install S\&C at every possible location (i.e. it is not the optimal design), even if we could afford the costs.

For the hypothetical Small and the Hearn networks, there is no significant difference between the quality of the solutions produced by the GH and the SA as shown in Fig. 5 (a) and (b); however, for the much larger Sioux Falls network, the SA produces better solutions and outperforms the GH with low budget (Fig. 5 (c)). As the budget increases, the difference between the two algorithms diminishes. However, the SA is still faster (Fig. 4 (c)).

Although optimally installing S\&C decreases the overall cost in transportation networks, it has different impacts on the three modes of transportation. For automobile and public transit modes, installing S\&C means building separate walkways for pedestrians, and it can decrease the travel time of automobile and public transit modes. However, for pedestrians, installing separate walkways can decrease crashes and 
therefore increase safety. For example, in the Sioux Falls network, as shown in Fig. 6, installing S\&C causes a larger reduction in walking travel costs than public transit and automobile costs. This can be explained by the effect that S\&C have on pedestrians’ safety (equation (4)).

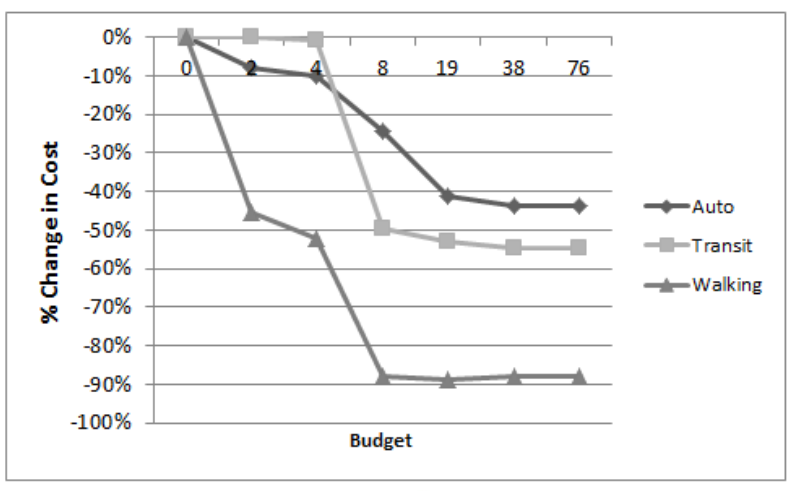

(a)

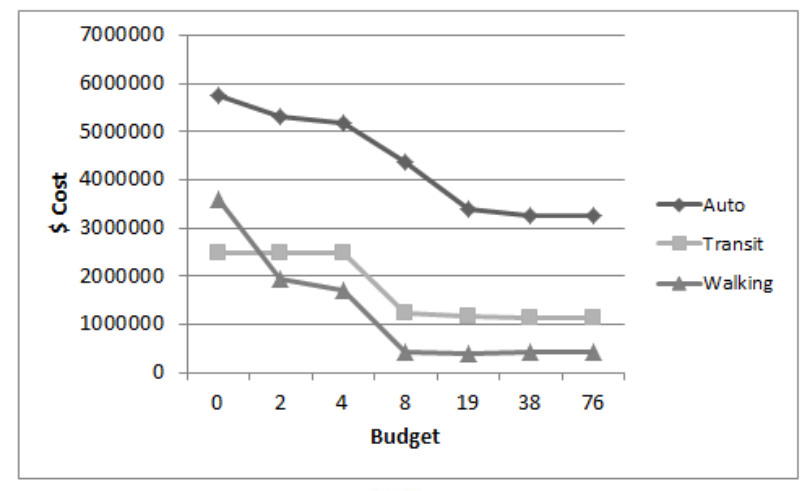

(b)

Fig.6. Changes in the overall transportation cost over different transportation modes for the Sioux Falls network: (a) Relative changes (percent of changes) in the overall cost, (b) Absolute changes in the overall cost.

\subsection{Sensitivity analysis: Demand}

To see the impact of installing S\&C on a transportation network under different demands, we conducted a sensitivity analysis (shown in Fig. 7). A multiplier was used for demand, called the "demand factor". Using the demand factor, we changed the demand in the networks from $0.25 \%$ of the default value up to $800 \%$.

The results show that when the networks are less crowded (i.e. under low traffic conditions), installing S\&C does not have significant impact on the overall transportation cost. As the demand increases and more people use the network, S\&C start to have more impact on the network until a certain point at which in the network becomes overcrowded and the impact of S\&C diminishes (Fig. 7 (a) and (b)) or remains unchanged (Fig. 7 (c)). 


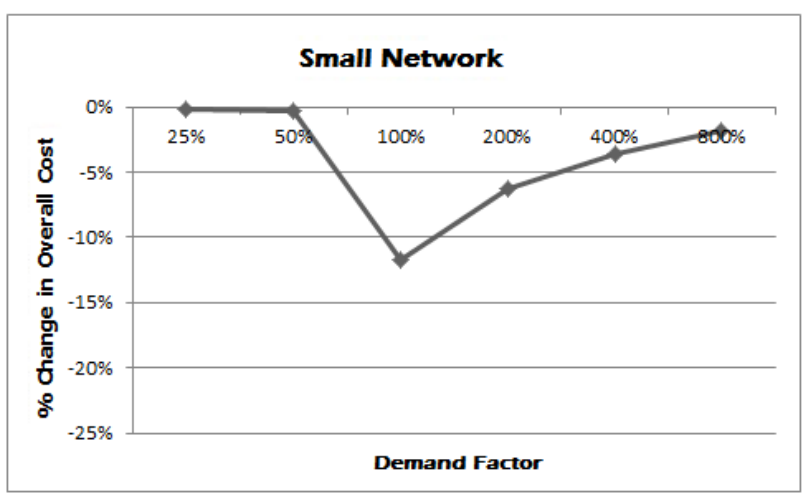

(a)

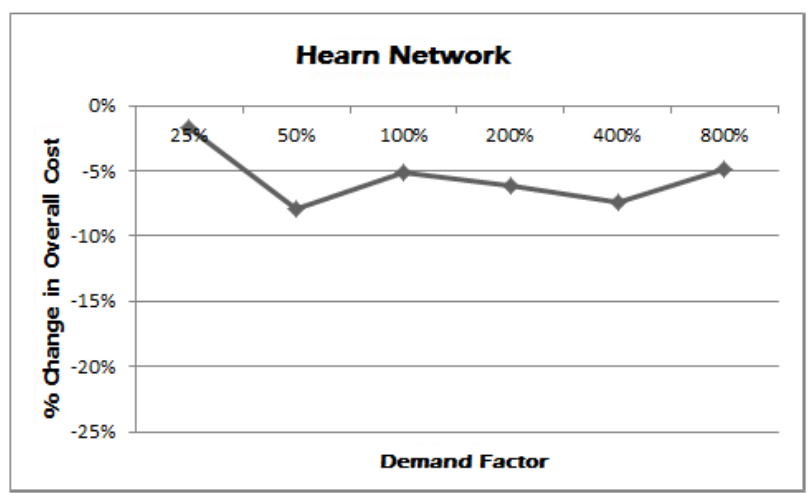

(b)

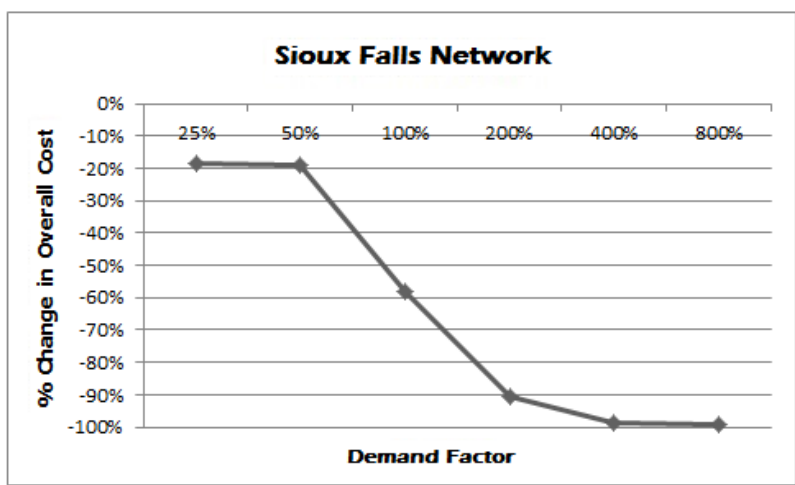

(c)

Fig. 7. Percent change in the overall cost due to traffic calming facilities for different demand factors for each OD pair: (a) Small network, (b) Hearn network, and (c) Sioux Falls network

\subsection{Sensitivity analysis: Safety weight factor}

In the travel cost function for sidewalk links (equation (4)), we used $\delta$ as a safety weight factor for trade-off between travel cost and safety. This safety weight factor is used to combine the travel time part and the safety part in the travel cost function of sidewalk links. To investigate the impact of safety on the overall cost in the networks, and to find a compromise between safety and time in the objective function, we performed another set of experiments by changing the safety weight factor's value in a range from 0 to 1. As shown in Fig. 8., for all values of the safety weight factor, installing S\&C decreases the overall cost in all three networks. As the value of the safety weight increases, so does the impact of S\&C on the overall cost (the overall transportation cost decreases more). This impact is more significant in the Sioux Falls network (see Fig. 8.). 


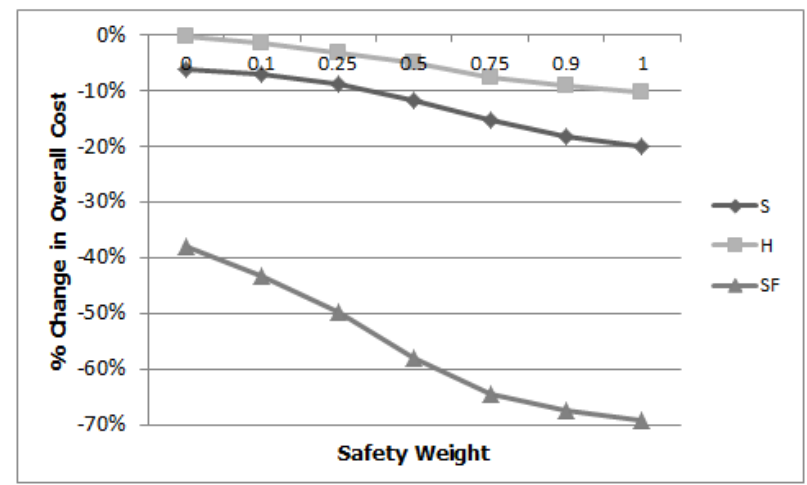

Fig.8. Percent change in the overall cost considering different values for the safety weight factor ( $\mathrm{S}=$ Small Network, H = Hearn Network, SF = Sioux Falls Network)

\subsection{Flow demonstration in the Small Network}

To see the impact of installing $\mathrm{S} \& \mathrm{C}$ on the traffic flow in transportation networks considering changes in demand and the safety weight factor, we conducted a series of experiments on the Small network. We investigated the travelers' flows in the network under three scenarios: (1) before and after installing S\&C (with a safety weight factor of $\delta=0.5$, and a demand factor of 100\%), (2) for low and high traffic congestion after installing S\&C (a low and high demand factor), and (3) for low and high values of the safety weight factor (after installing $\mathrm{S} \& \mathrm{C}$ ).

Figs. 9.1.a. and Fig. 9.1.b. respectively show the flows in the network before and after installing S\&C. In general, we observed that after installing $\mathrm{S} \& \mathrm{C}$, fewer automobiles and more walking are used. More specifically, the automobile link $(1,3)$ is no longer used after installing $\mathrm{S} \& \mathrm{C}$, and the unused public transit link $(1001,3001)$ is used after installing S\&C. Some walking links are also used more often after installing S\&C, such as $(2,324),(324,431)$, and $(431,413)$. The public transit link $(3001,4001)$ is used less often after installing S\&C.

Figures 9.2.a and 9.2.b. show the difference for the flow in the Small network after installing S\&C under low and high traffic congestion (low and high demand factor) respectively. The model suggests that when the transportation network becomes more crowded, fewer automobiles and more walking and public transit are used. This result is expected: when there is high automobile traffic congestion, people are more likely to walk than to use their own vehicles because automobile traffic congestion causes slow travel speeds. 


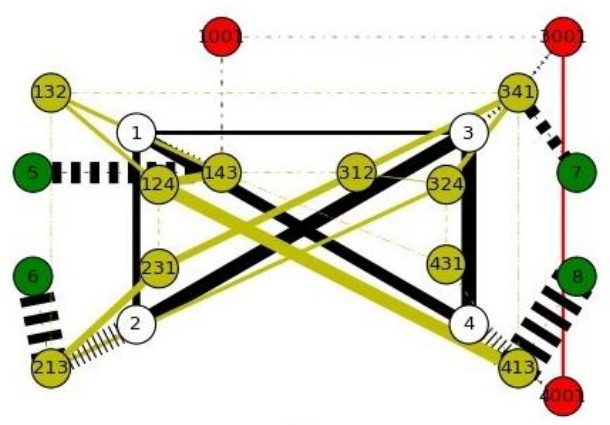

1.a.

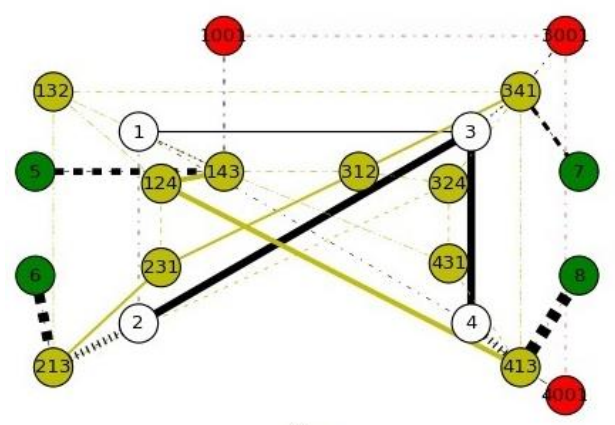

2.a.

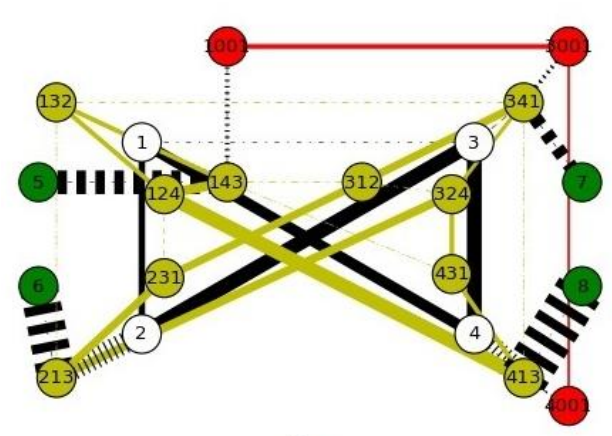

3.a.

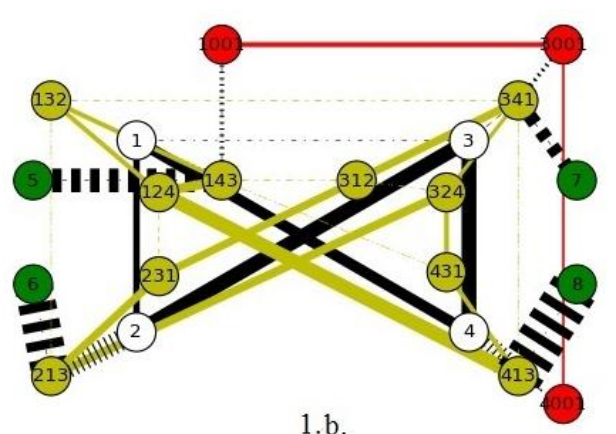

1.b.
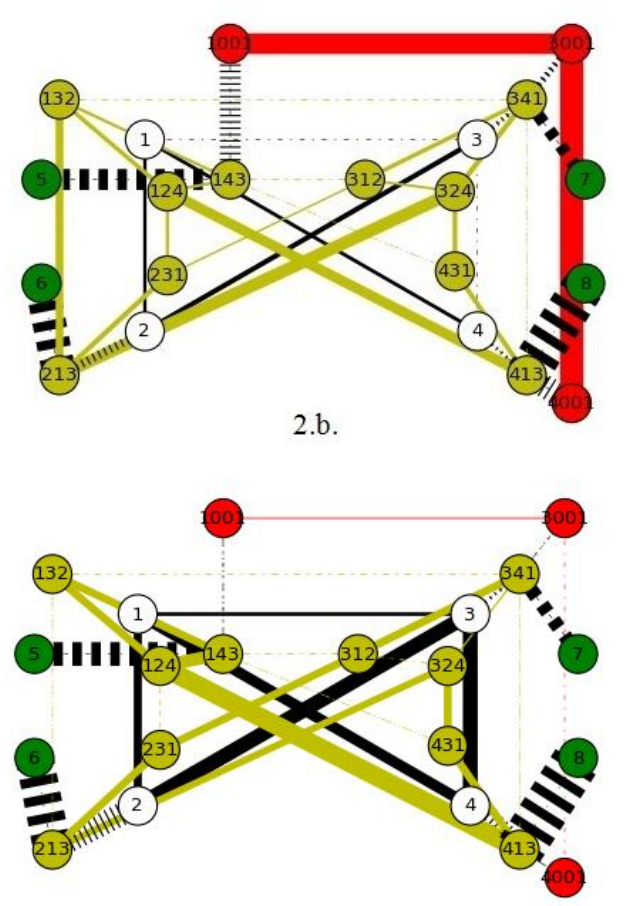

3.b.

Fig.9. The flow in the Small Transportation Network under different scenarios: 1. before (a) and after (b) implementing traffic calming facilities, 2. for low (a) and high (b) crowd congestion, and 3. for low (a) and high (b) safety weight factors.

Figures 9.3.a and 9.3.b. show the travelers' flow in the Small transportation network for low safety weight $(\delta=0.1)$ and high safety weight $(\delta=0.9)$. As shown in Figs. 9.3.a and 9.3.b, the automobile links $(1,3)$ and $(2,1)$ and the walking links $(124,413),(143,124),(132,124),(324,431)$, and $(431,413)$ are used more often for a higher value of the safety weight factor. On the other hand, public transit links $(1001,3001)$ and $(3001,4001)$ are used less often. We speculate that increasing the safety weight factor (and therefore putting more emphasis on pedestrians' safety) leads to more use of walking and automobile links and less use of the public transit mode. The presence of safe walkways that are separate from roads can encourage more people to walk. As a result, the flow of pedestrians would cause less disturbance to 
the flow of automobile, which can decrease the travel time for automobile, increase the use of automobile and decrease that of public transit.

\section{Conclusion}

This study provides insights into how implementing traffic calming facilities such as sidewalks and crosswalks (S\&C) affects pedestrians' safety and the transportation cost in a multimodal transportation network. This study considers pedestrians' safety as an important factor in designing a transportation network. A mixed-integer nonlinear programming model is developed for optimally locating S\&C in a transportation network. The model is implemented in YALMIP (version 20141030) and solved using the BARON solver (version v1.69), one of the most advanced solvers on the market. However, because of the computational difficulty for the YALMIP/BARON MINLP formulation, two heuristic methods, a customized Greedy Heuristic and a Simulated Annealing algorithm, are developed for the problem. Experiments with three sample networks show that these algorithms outperform the BARON solver. Specifically, the SA algorithm is more efficient in producing better quality solutions for the Sioux Falls network (the largest sample network). Although these two approximate algorithms do not necessarily produce optimal solutions (i.e. the optimal design of a transportation network), the results they produce can help in better understanding the impact of traffic calming facilities (in this study, S\&C) in multimodal transportation networks. The results show that installing S\&C according to the solution obtained by the SA algorithm reduces the total transportation cost by $12 \%, 5 \%$ and 59\% respectively for the Small, Hearn and Sioux Falls networks. The optimal solutions are expected to yield an even greater reduction in transportation cost.

The major contribution of this research is to develop a quantitative network design model for locating traffic calming facilities in the transportation network, so as to reduce the overall cost and to improve pedestrians' safety. The results suggest that not only does installing S\&C improve pedestrians' safety, but it also reduces the total transportation cost (including the travel cost of automobile and public transit). Installing S\&C can provide separate walkways for pedestrians; as a result, pedestrians no longer have to walk along busy streets and interfere with automobile and public transit's traffic. This can reduce the number of car accidents that involve hitting a pedestrian and improve pedestrians' safety. It also can decrease the effect of pedestrian traffic on automobile and public transit modes, which in turn reduces the travel cost for automobile and public transit. However, S\&C provide a smaller reduction of the total transportation cost in a network with low traffic. The results also suggest that installing S\&C as safe walkways for pedestrians can encourage walking. This effect is consistent with the results of previous studies (e.g. (Gallimore et al., 2011; Pucher and Dijkstra, 2003; Southworth, 2005; Staunton et al., 2003)). More walking and less use of cars can improve public health (Lee and Buchner, 2008), alleviate traffic 
congestion, cut energy use and carbon emissions and reduce noise and air pollution (Marshall and Garrick, 2010; Pucher and Dijkstra, 2003; Southworth, 2005).

This study can be viewed as a foundation for further research on pedestrian transportation. Future research can be conducted in several directions. First, the only traffic calming facilities considered in this paper are sidewalks and crosswalks. However, there are many more to consider such as speed bumps, stop lights, stop signs and police patrols. On the other hand, in this study we only considered the safety effects of sidewalks (through equation (4)); one can also study the effect of crosswalks on pedestrians' safety. We assumed that the cost of installing S\&C at any location in a transportation network is the same. This assumption can be relaxed by acquiring relevant data. Although safety is an important factor in promoting walking, connectivity is also important in designing a walkable transportation system (Southworth, 2005). Restrictions can be added to ensure connectivity when designing walkways. The transportation modes we considered in this study are walking, automobile, and public transit, but one can consider other transportation modes such as bicycles. Further, the problem we studied in this paper is deterministic. Therefore, considering uncertainty (in demand, capacity, travel time, etc.) is another way to extend this research. Regarding the solution methodology, since the heuristic methods used in this study produce approximate solutions, a useful next step is to develop exact methods. As the problem is bi-level in nature, implementing a decomposition-based method is recommended.

\section{Acknowledgement}

This work was supported by the National Center for Intermodal Transportation for Economic Competitiveness (NCITEC) at Mississippi State University. 


\section{Appendix}

Proof of Proposition 1: We will show that the objective function of the lower level problem, $\varphi_{l, m}($.$) , is a$ combination of convex functions and therefore convex. First we will show that the objective function for the automobile link, $\varphi_{l, a}($.$) , (equation (9)) is convex. If we ignore \vartheta$ and $\mu_{l a}$ in (9) (as they are positive constant values), and denote $x_{l m}=\sum_{k \in K} X_{k l m}$ for all links $(l, m)$, we can rewrite (9) as:

$$
\begin{gathered}
\varphi_{l, a}(X, Y)=\frac{1}{\left(\gamma_{l, s}\right)^{\beta_{1}}}\left(x_{l, s 1}^{\beta_{1}}+x_{l, s 2} \beta_{1}\right)+\frac{1}{\left(\gamma_{l, c}\right)^{\beta_{2}}}\left(x_{l, b x} \beta_{2}+x_{l, e x} \beta_{2}\right) \\
+t_{l a}\left(1+\alpha_{1}\left(\frac{x_{l a}+\omega x_{l, t}}{\gamma_{l a}}\right)^{\beta_{3}}\right)+\mu_{l, a}
\end{gathered}
$$

As $x^{a}$ is convex for $a \geq 1$ or $a<0$, therefore, for $\beta_{1}, \beta_{2} \geq 1$ or $\beta_{1}, \beta_{2}<0$ the first line (parts related to $\mathrm{S} \& \mathrm{C}$ ) of (16) is convex. For simplicity we assume $\sum_{k \in K} X_{k l a}=x$ and $\sum_{k \in K} X_{k, \mathrm{l}, \mathrm{t}}=y$; then, the Hessian matrix of the last part in equation (16) (the second line) is:

$$
H=\frac{1}{\gamma_{l a}^{2}}\left[\begin{array}{cc}
\beta_{3}\left(\beta_{3}-1\right) \alpha_{1} t_{l a}\left(\frac{x+\omega y}{\gamma_{l a}}\right)^{\beta_{3}-2} & \beta_{3}\left(\beta_{3}-1\right) \alpha_{1} t_{l a} \omega\left(\frac{x+\omega y}{\gamma_{l a}}\right)^{\beta_{3}-2} \\
\beta_{3}\left(\beta_{3}-1\right) \alpha_{1} t_{l a} \omega\left(\frac{x+\omega y}{\gamma_{l a}}\right)^{\beta_{3}-2} & \beta_{3}\left(\beta_{3}-1\right) \alpha_{1} t_{l a} \omega^{2}\left(\frac{x+\omega y}{\gamma_{l a}}\right)^{\beta_{3}-2}
\end{array}\right]
$$

A $n \times n$ real symmetric matrix $M$ is positive semi-definite if $z^{T} \times M \times z \geq 0$ for all non-zero vectors $z$ with real entries. For an arbitrary non-zero vector $z=\left[\begin{array}{ll}a & b\end{array}\right]$, if we show that $\mathrm{z}^{\mathrm{T}} \times \mathrm{H} \times \mathrm{z} \geq$ 0 , then $H$ is positive semidefinite.

$$
\begin{aligned}
& z^{T} \times H \times z=\left[a \beta_{3}\left(\beta_{3}-1\right) \alpha_{1} t_{l a}\left(\frac{x+\omega y}{\gamma_{l a}}\right)^{\beta_{3}-2}+b \beta_{3}\left(\beta_{3}-1\right) \alpha_{1} t_{l a} \omega\left(\frac{x+\omega y}{\gamma_{l a}}\right)^{\beta_{3}-2}\right. \\
& \left.\mathrm{a} \beta_{3}\left(\beta_{3}-1\right) \alpha_{1} t_{l a} \omega\left(\frac{x+\omega y}{\gamma_{l a}}\right)^{\beta_{3}-2}+b \beta_{3}\left(\beta_{3}-1\right) \alpha_{1} t_{l a} \omega^{2}\left(\frac{x+\omega y}{\gamma_{l a}}\right)^{\beta_{3}-2}\right] \times\left[\begin{array}{l}
a \\
b
\end{array}\right]= \\
& a^{2} \beta_{3}\left(\beta_{3}-1\right) \alpha_{1} t_{l a}\left(\frac{x+\omega y}{\gamma_{l a}}\right)^{\beta_{3}-2}+a b \beta_{3}\left(\beta_{3}-1\right) \alpha_{1} t_{l a} \omega\left(\frac{x+\omega y}{\gamma_{l a}}\right)^{\beta_{3}-2}+b \mathrm{a} \beta_{3}\left(\beta_{3}-1\right) \alpha_{1} t_{l a} \omega\left(\frac{x+\omega y}{\gamma_{l a}}\right)^{\beta_{3}-2}+ \\
& b^{2} \beta_{3}\left(\beta_{3}-1\right) \alpha_{1} t_{l a} \omega^{2}\left(\frac{x+\omega y}{\gamma_{l a}}\right)^{\beta_{3}-2}
\end{aligned}
$$

We need to show that the above expression is non-negative:

$$
\begin{aligned}
& a^{2} \beta_{3}\left(\beta_{3}-1\right) \alpha_{1} t_{l a}\left(\frac{x+\omega y}{\gamma_{l a}}\right)^{\beta_{3}-2}+2 a b \beta_{3}\left(\beta_{3}-1\right) \alpha_{1} t_{l a} \omega\left(\frac{x+\omega y}{\gamma_{l a}}\right)^{\beta_{3}-2}+b^{2} \beta_{3}\left(\beta_{3}-1\right) \alpha_{1} t_{l a} \omega^{2}\left(\frac{x+\omega y}{\gamma_{l a}}\right)^{\beta_{3}-2} \\
& =\beta_{3}\left(\beta_{3}-1\right) \alpha_{1} t_{l a}\left(\frac{x+\omega y}{\gamma_{l a}}\right)^{\beta_{3}-2}\left(a^{2}+2 a b \omega+b^{2} \omega^{2}\right)
\end{aligned}
$$


$=\beta_{3}\left(\beta_{3}-1\right) \alpha_{1} t_{l a}\left(\frac{x+\omega y}{\gamma_{l a}}\right)^{\beta_{3}-2}(a+b \omega)^{2}$

Knowing that $x, y, t_{l a}, \omega$ and $\gamma_{l a}$ are all positive, for $\beta_{3} \geq 1$ or $\beta_{3}<0$, the Hessian will always be nonnegative, and therefore $\varphi_{l, a}($.$) (represented by equation (9)) in the objective function is convex. In a$ similar way we can show that equations (10) and (13) are also convex. For $\beta_{5} \geq 1$ or $\beta_{5}<0$ and positive values of $\propto_{3}, \gamma_{l p}$ and $\sigma$, equation (11) is convex as long as $P_{l a}\left(X_{l s}\right) X_{l s}$ is convex. In our case, $P_{l a}\left(X_{l s}\right)$ is a linear expression with positive coefficients, and therefore $P_{l a}\left(X_{l s}\right) X_{l s}$ is convex. Equation (14) consists of positive constant parameters and equation (15) is zero. Thus, the objective function in the lower level problem is a sum of convex expressions, which indicates that it is convex.

\section{References}

Aashtiani, 1979. The multi-modal traffic assignment problem (Doctoral dissertation). Massachusetts Institute of Technology.

Aliakbarian, N., Dehghanian, F., Salari, M., 2015. A bi-level programming model for protection of hierarchical facilities under imminent attacks. Comput. Oper. Res. 64, 210-224.

Bahari, N.I., Arshad, A.K., Yahya, Z., 2013. Assessing the pedestrians' perception of the sidewalk facilities based on pedestrian travel purpose, in: 2013 IEEE 9th International Colloquium on Signal Processing and Its Applications. pp. 27-32.

Beltran, B., Carrese, S., Cipriani, E., Petrelli, M., 2009. Transit network design with allocation of green vehicles: A genetic algorithm approach. Transp. Res. Part C Emerg. Technol. 17, 475-483.

Ben-Ayed, O., Blair, C.E., 1990. Computational difficulties of bilevel linear programming. Oper. Res. 38, 556-560.

Bouleimen, K., Lecocq, H., 2003. A new efficient simulated annealing algorithm for the resource-constrained project scheduling problem and its multiple mode version. Eur. J. Oper. Res. 149, 268-281.

Buehler, R., Pucher, J., 2012. Walking and cycling in Western Europe and the United States: trends, policies, and lessons. TR News.

Campbell, B.J., Zegeer, C. V, Huang, H.H., Cynecki, M.J., 2004. A Review of Pedestrian Safety Research in the United States and Abroad.

Cantarella, G.E., Pavone, G., Vitetta, A., 2006. Heuristics for urban road network design: Lane layout and signal settings. Eur. J. Oper. Res. 175, 1682-1695.

Cipriani, E., Petrelli, M., Fusco, G., 2006. A multimodal transit network design procedure for urban areas. Adv. Transp. Stud. 10.

Drezner, Z., Salhi, S., 2002. Using hybrid metaheuristics for the one-way and two-way network design problem. Nav. Res. Logist. 49, 449-463.

Fan, W., Machemehl, R.B., 2008. Tabu search strategies for the public transportation network optimizations with variable transit demand. Comput. Civ. Infrastruct. Eng. 23, 502-520.

Farahani, R.Z., Miandoabchi, E., Szeto, W.Y., Rashidi, H., 2013. A review of urban transportation network design problems. Eur. J. Oper. Res. 229, 281-302.

Farvaresh, H., Sepehri, M.M., 2013. A branch and bound algorithm for bi-level discrete network design problem. Networks Spat. Econ. 13, 67-106.

Farvaresh, H., Sepehri, M.M., 2011. A single-level mixed integer linear formulation for a bi-level discrete network 
design problem. Transp. Res. Part E Logist. Transp. Rev. 47, 623-640.

FHWA, 2010. Safety Benefits of Walkways, Sidewalks, and Paved Shoulders. Technical report, U.S. Dept. of Transportation, Federal Highway Administration, Office of Safety, Washington, D.C. [WWW Document]. URL http://safety.fhwa.dot.gov/ped_bike/tools_solve/walkways_trifold/ (accessed 4.22.14).

FHWA, ITE, 1999. Traffic Calming: State of the Practice. Report by Institute of Transportation Engineers [WWW Document]. Inst. Transp. Eng. URL http://www.ite.org/traffic/tcstate.asp

Frank, M., Wolfe, P., 1956. An algorithm for quadratic programming. Nav. Res. Logist. Q. 3, 95-110.

Freeman, L., Neckerman, K., Schwartz-Soicher, O., Quinn, J., Richards, C., Bader, M.D.M., Lovasi, G., Jack, D., Weiss, C., Konty, K., others, 2013. Neighborhood walkability and active travel (walking and cycling) in New York City. J. Urban Heal. 90, 575-585.

Friederichs, S.A., Kremers, S.P., Lechner, L., de Vries, N.K., 2013. Neighborhood walkability and walking behavior: the moderating role of action orientation. J Phys Act Heal. 10, 15-22.

Gallimore, J.M., Brown, B.B., Werner, C.M., 2011. Walking routes to school in new urban and suburban neighborhoods: An environmental walkability analysis of blocks and routes. J. Environ. Psychol. 31, 184-191.

Gallo, M., Montella, B., D'Acierno, L., 2011. The transit network design problem with elastic demand and internalisation of external costs: An application to rail frequency optimisation. Transp. Res. Part C Emerg. Technol. 19, 1276-1305.

Gårder, P.E., 2004. The impact of speed and other variables on pedestrian safety in Maine. Accid. Anal. Prev. 36, 533-542.

Hearn, D.W., Ramana, M. V., 1998. Solving congestion toll pricing models. Springer US.

Khooban, Z., Farahani, R.Z., Miandoabchi, E., Szeto, W.Y., 2015. Mixed network design using hybrid scatter search. Eur. J. Oper. Res. 247, 699-710.

Kia, R., Baboli, A., Javadian, N., Tavakkoli-Moghaddam, R., Kazemi, M., Khorrami, J., 2012. Solving a group layout design model of a dynamic cellular manufacturing system with alternative process routings, lot splitting and flexible reconfiguration by simulated annealing. Comput. Oper. Res. 39, 2642-2658.

Kirkpatrick, S., Vecchi, M.P., 1983. Optimization by simulated annealing. Science (80-. ). 220, 671-680.

LeBlanc, L.J., 1975. An algorithm for the discrete network design problem. Transp. Sci. 9, 183-199.

Lee, G., Joo, S., Oh, C., Choi, K., 2013. An evaluation framework for traffic calming measures in residential areas. Transp. Res. Part D Transp. Environ. 25, 68-76.

Lee, I.-M., Buchner, D.M., 2008. The importance of walking to public health. Med. Sci. Sports Exerc. 40, S512-8.

Lofberg, J., 2004. YALMIP: A toolbox for modeling and optimization in MATLAB, in: Computer Aided Control Systems Design, 2004 IEEE International Symposium on. pp. 284-289.

Madadi, A., Kurz, M.E., Mason, S.J., Taaffe, K.M., 2014. Supply chain design under quality disruptions and tainted materials delivery. Transp. Res. Part E Logist. Transp. Rev. 67, 105-123.

Marshall, W.E., Garrick, N.W., 2010. Effect of street network design on walking and biking. Transp. Res. Rec. J. Transp. Res. Board 2198, 103-115.

Mesbah, M., Sarvi, M., Currie, G., 2008. New methodology for optimizing transit priority at the network level. Transp. Res. Rec. J. Transp. Res. Board 2089, 93-100.

Miandoabchi, E., Daneshzand, F., Szeto, W.Y., Farahani, R.Z., 2013. Multi-objective discrete urban road network design. Comput. Oper. Res. 40, 2429-2449.

Miandoabchi, E., Farahani, R.Z., Dullaert, W., Szeto, W.Y., 2012. Hybrid evolutionary metaheuristics for concurrent multi-objective design of urban road and public transit networks. Networks Spat. Econ. 12, 441480. 
Miandoabchi, E., Farahani, R.Z., Dullaert, W., Szeto, W.Y., 2011a. Hybrid Evolutionary Metaheuristics for Concurrent Multi-Objective Design of Urban Road and Public Transit Networks. Networks Spat. Econ. 12, $441-480$.

Miandoabchi, E., Farahani, R.Z., Szeto, W.Y., 2011b. Bi-objective bimodal urban road network design using hybrid metaheuristics. Cent. Eur. J. Oper. Res. 20, 583-621.

Millward, H., Spinney, J., Scott, D., 2013. Active-transport walking behavior: destinations, durations, distances. J. Transp. Geogr. 28, 101-110.

Nadesan-Reddy, N., Knight, S., 2013. The effect of traffic calming on pedestrian injuries and motor vehicle collisions in two areas of the eThekwini Municipality: A before-and-after study. SAMJ South African Med. J. $103,621-625$.

Parsafard, M., Rashidi, E., Li, X., Medal, H., 2015. A Multimodal User Equilibrium Traffic Assignment with Network Reconstruction.

Prokopich, W., Wise, J., 2014. Traffic Calming and RRFBs Increase Pedestrian Safety in Lethbridge, Canada. IMSA J. 52.

Pucher, J., Dijkstra, L., 2003. Promoting safe walking and cycling to improve public health: lessons from the Netherlands and Germany. Am. J. Public Health 93, 1509-1516.

Resat, H.G., Turkay, M., 2015. Design and operation of intermodal transportation network in the Marmara region of Turkey. Transp. Res. Part E Logist. Transp. Rev. 83, 16-33.

Sahinidis, N. V., 2014. BARON 14.3.1: Global Optimization of Mixed-Integer Nonlinear Programs, User's Manual.

Seo, Y.U., Park, J.H., Jang, H., Lee, Y.I., 2005. A study on setting-up a methodology and criterion of exclusive bus lane in urban area, in: Proceedings of the Eastern Asia Society for Transportation Studies. pp. 339-351.

Southworth, M., 2005. Designing the walkable city. J. urban Plan. Dev. 131, 246-257.

Staunton, C.E., Hubsmith, D., Kallins, W., 2003. Promoting safe walking and biking to school: the Marin County success story. Am. J. Public Health 93, 1431-1434.

Stewart, O.T., Moudon, A.V., Saelens, B.E., Lee, C., Kang, B., Doescher, M.P., 2016. Comparing Associations Between the Built Environment and Walking in Rural Small Towns and a Large Metropolitan Area. Environ. Behav. 48, 13-36.

Szeto, W.Y., Jaber, X., OMahony, M., 2010. Time-Dependent Discrete Network Design Frameworks Considering Land Use. Comput. Civ. Infrastruct. Eng. 25, 411-426.

Szeto, W.Y., Jiang, Y., 2014. Transit route and frequency design: Bi-level modeling and hybrid artificial bee colony algorithm approach. Transp. Res. Part B Methodol. 67, 235-263.

Toobaie, S., Aashtiani, H.Z., Hamedi, M., Haghani, A., 2010. Application of a complementarity approach to large scale traffic equilibrium problems, in: Transportation Research Board. pp. 1-29.

Vandervalk, A., Louch, H., Guerre, J., Margiotta, R., 2014. Incorporating Reliability Performance Measures Into the Transportation Planning and Programming Processes: Technical Reference.

Weinstein Agrawal, A., Schlossberg, M., Irvin, K., 2008. How Far, by Which Route and Why? A Spatial Analysis of Pedestrian Preference. J. Urban Des. 13, 81-98.

Yamada, T., Febri, Z., 2015. Freight transport network design using particle swarm optimisation in supply chain-transport supernetwork equilibrium. Transp. Res. Part E Logist. Transp. Rev. 75, 164-187.

Yang, Z., Yu, B., Cheng, C., 2007. A Parallel Ant Colony Algorithm for Bus Network Optimization. Comput. Civ. Infrastruct. Eng. 22, 44-55.

Yao, B., Hu, P., Lu, X., Gao, J., Zhang, M., 2014. Transit network design based on travel time reliability. Transp. Res. Part C Emerg. Technol. 43, 233-248. 
Yu, B., Kong, L., Sun, Y., Yao, B., Gao, Z., 2015. A bi-level programming for bus lane network design. Transp. Res. Part C Emerg. Technol. 55, 310-327. 